\title{
MÉTODOS E TÉCNICAS GEOELÉTRICAS RASAS NA DELIMITAÇÃO DE ÁREA AFETADA POR RUPTURA EM TETO DE TÚNEL URBANO
}

\author{
José D. F. Gallas ${ }^{1}$, Fabio Taioli ${ }^{1}$, Walter Malagutti Filho ${ }^{2}$, Renato L. Prado ${ }^{1}$ \& João C. \\ Dourado $^{2}$
}

Received June 06, 2001 / Accepted March 15, 2002

\begin{abstract}
Este trabalho apresenta um uso da geofísica aplicada em uma situação de ambiente urbano. O objetivo dos levantamentos foi o de delimitar a extensão da zona afetada por um colapso e desmoronamento do teto de um túnel em construção sob o Parque do Ibirapuera na capital paulista. São descritos e apresentados os resultados obtidos através do emprego dos métodos geofísicos geoelétricos e suas técnicas, em uma situação emergencial e de risco em uma área urbana na cidade de São Paulo. Também são comparadas as técnicas/arranjos de campo e a forma de apresentação e interpretação dos mesmos. Os métodos empregados foram a Eletrorresistividade, Polarização Induzida (IP) e Potencial Espontâneo (SP). Os trabalhos de campo e interpretação preliminar foram efetuados em apwenas um fim de semana (sábado e domingo). Os resultados mostraram-se plenamente satisfatórios e possibilitaram significativa redução nos custos nas obras de remediação da área do incidente, cuja área decresceu da inicialmente estimada pela engenharia $(20 \times 20 \mathrm{~m})$ para $3 \times 5 \mathrm{~m}$ definida pelos estudos geofísicos.
\end{abstract}

Palavras-chave: Geofísica aplicada; Geofísica urbana; Bacia sedimentar de São Paulo.

SHALLOW GEOELECTRIC METHODS AND TECHNIQUES TO DEFINE AN AREA AFFECTED BY ROOF FAILURE OF AN URBAN TUNNEL - This paper presents a case study where geophysics was successfully applied to solve a complex problem associated with a tunnel excavation accident in an urban area. It presents and describes the results obtained with geoelectrical methods applied in an emergencial task in a risky urban area in São Paulo City. It also compares the techniques and field arrays, ways to present and interpret the data. The employed methods were electroresistivity, induced polarization (IP) and self potential (SP). The objective of the survey was to delineate the extension of a roof failure of an urban tunnel under excavation beneath the Ibirapuera Park in order to orientate the volume of tunnel roof should be treated. Only two days (Saturday and Sunday) were available to carry out the survey and its interpretation. The results were considered satisfactory and brought a significant economy to the final cost of the tunnel. This economy was due to the decreasing of the remediation area from the initial $20 \times 20 \mathrm{~m}$ (estimated by the engineers) to $3 \times 5 \mathrm{~m}$ (estimated by the geophysical survey).

Key words: Applied geophysics; Urban geophysics; São Paulo Basin.






\section{INTRODUÇÃO E HISTÓRICO}

A escavação de túneis em áreas urbanas envolve elevados custos e as dificuldades inerentes a este ambiente. Mesmo as investigações através de sondagens são difíceis, havendo dificuldades de transporte e instalação dos equipamentos, sob os aspectos de acesso e espaços físicos.

Considerando investigações indiretas, como é o caso da geofísica aplicada, as dificuldades tornam-se ainda maiores. Além dos empecilhos encontrados nas sondagens mecânicas, têm-se, nestas situações, a presença de ruídos (elétricos, eletromagnéticos, vibrações, principalmente) que interferem enormemente nas propriedades físicas medidas.

Estes são os principais motivos de não serem comuns os casos, citados na bibliografia, que tratam da geofísica aplicada em situações urbanas. Resultados de aplicações geofísicas em trabalhos similares foram obtidos por Gallas \& Augusto Filho (1999) e Gallas (1999), em que é tratado um caso de área cárstica. Um caso de aplicação de métodos geolétricos subsidiando o diagnóstico de recalques em terrenos colapsíveis é o enfoque dado por Gallas et al. (2000).

Durante a escavação do túnel sob o Parque do Ibirapuera, cidade de São Paulo, houve colapso de uma parte do teto do túnel, com desmoronamento do estrato sobrejacente. O local onde ocorreu o acidente foi na porção onde o túnel estava sendo alargado para construção de área de estacionamento de emergência (baia).

Na ocasião em que os trabalhos geofísicos foram efetuados, algumas providências emergenciais haviam sido tomadas, tais como aplicação de concreto projetado, injeção de aproximadamente $15 \mathrm{~m}^{3}$ de calda de cimento e também escoramento do teto com o braço de uma escavadeira.

Segundo informações obtidas no local da obra, houve uma primeira ruptura e conseqüente desmoronamento durante a instalação da primeira cambota metálica do trecho alargado. Uma repetição do acidente ocorreu quando da escavação para instalação da segunda cambota, tendo a ruptura principal acontecido durante os trabalhos para a colocação da quarta cambota metálica.

De acordo com estimativas efetuadas por técnicos no local do incidente, avaliou-se que cerca de $20 \mathrm{~m}^{3}$ de material desabou do teto do túnel. O material desmoronado era constituído essencialmente por areia fina siltosa que, misturada com muita água, fluiu em forma de lama nos primeiros momentos do incidente principal. Esquematicamente, a Fig. 1 mostra que o desmoronamento ocorreu exatamente na passagem, durante a escavação do túnel, da argilla muito rija (Standard Penetration Test - SPT $>30$ ) e penetra no nível de areia fina siltosa (SPT 4 a 15).

Os levantamentos geofísicos foram solicitados em caráter emergencial pela Empresa Municipal de Urbanização - EMURB e pelas empresas contratadas para execução da obra, imediatamente após ocorrido o incidente, visando estimar a área do maciço que deveria ser reforçada com tratamento especial (jetcret), inclusive para se ter uma avaliação dos custos da remediação do local. A estimativa inicial era de que a área atingida seria da ordem de $20 \times 20 \mathrm{~m}$, o que representaria custos significativamente elevados. Após a interpretação dos dados geofísicos e confirmação direta a seguir, verificou-se que a área afetada era bastante menor, proporcionando uma redução de custos superior a $90 \%$ da avaliação preliminar.

Os ensaios foram realizados em um final de semana pelo Laboratório de Geofísica Aplicada do Instituto de Pesquisas Tecnológicas do Estado de São Paulo - IPT e pelo Instituto de Geociências da USP em decorrência do desplacamento de parte do teto do túnel sob o Parque do Ibirapuera na cidade de São Paulo. Este túnel é o que atualmente interliga as Avenidas Juscelino Kubitschek, Sena Madureira e 23 de Maio.

Os levantamentos geofísicos consistiram de polarização induzida (IP), eletrorresistividade, potencial espontâneo (SP) e sísmica de reflexão. Neste trabalho são apresentados os resultados e as interpretações referentes aos 3 primeiros métodos. Os dados sísmicos foram apresentados em Taioli et al. (1997a).

\section{GEOLOGIA-GEOTECNIA}

A região onde foram realizados os ensaios geofísicos é constituída fundamentalmente por sedimentos pertencentes à Bacia de São Paulo, localmente sob uma cobertura de aterro.

Segundo Rebouças et al. (1983) e Riccomini (1989) estes sedimentos integram a Formação Resende. Os materiais descritos, oriundos de investigações mecânicas, coincidem com os trabalhos realizados por Takiya (1991) e Cozzolino et al. (1994) em locais da Bacia de São Paulo.

A Fig. 1 mostra a seção geológica-geotécnica simplificada do local e a posição onde situa-se a escavação do túnel. 
No local dos trabalhos tem-se inicialmente uma camada de aterro com espessura variando entre 1 a $2 \mathrm{~m}$ (entre cotas 756 e $754 \mathrm{~m}$ ) constituída de argila siltoarenosa de cor marrom avermelhada com valores de SPT da ordem de 4 golpes.

Logo a seguir são encontradas as camadas da Bacia sedimentar de São Paulo, de idade terciária. O primeiro estrato destes sedimentos é uma camada de argila amarelada mole com SPT em torno de 2 golpes e espessura de aproximadamente $2 \mathrm{~m}$, verificada através de sondagens.

Abaixo desta encontra-se uma camada com cerca de $8 \mathrm{~m}$ de argila rija de cor cinzenta, com SPT entre 15 e 20 golpes. $O$ contato entre esta e a camada superior situa-se ao redor da cota $753 \mathrm{~m}$. Dentro desta camada é comum a ocorrência de lentes de areia fina siltosa



Figura 1 - Seção geológico-geotécnica e posição do túnel na mesma.

Figure 1 - Geological-geotechnical section and tunnel location.

com SPT mais baixo (entre 7 e 10), podendo estas lentes conter cascalhos de tamanhos variados.

Na seqüência ocorre uma camada de argila fina siltosa gradando localizadamente a média e grossa em sua base, tendo espessura em torno de $5 \mathrm{~m}$. Seu topo está entre as cotas 745 e $746 \mathrm{~m}$ e a base ao redor da cota $741 \mathrm{~m}$, sendo o SPT entre 4 e 15 .

Na base do pacote de sedimentos, ocorre uma camada de argila muito rija de cor cinzenta e eventualmente variegada com SPT maior que 30 golpes. É nesta camada, em geral, que o túnel é escavado.

\section{CONSIDERAÇÕES TEÓRICAS SOBRE OS MÉTODOS E TÉCNICAS UTILIZADOS}

A prospecção geofísica trata da detecção em subsuperfície de corpos ou estruturas geológicas que apresentem algum tipo de contraste em uma ou mais propriedades físicas com o meio circundante e que possam ser medidas, na maioria dos casos, na superfície do terreno. Os métodos geofísicos aqui enfocados situam-se entre os Métodos Geoelétricos e podem ser subdivididos em Métodos de Campo Natural e Métodos de Campo Artificial, sendo os seguintes:

- Método do Potencial Espontâneo (campo natural)

- Método da Eletrorresistividade (campo artificial)

- Método da Polarização Induzida (campo artificial)

Os métodos geofísicos empregados neste trabalho, bem como técnicas de campo e suas aplicações práticas nas áreas de prospecção mineral, hidrogeologia, geologia de engenharia e geologia ambiental são discutidos e abordados em detalhe por Gallas (2000).

\section{POTENCIAL ESPONTÂNEO}

A expressão inglesa "Self Potential" originou a sigla SP e é assim como é referido este método no meio técnico.

Trata-se de um método de campo natural e baseiase no fato de que mesmo na ausência de qualquer campo elétrico criado artificialmente, é possível medir uma diferença de potencial entre dois eletrodos impolarizáveis introduzidos no terreno, que pode ser de alguns milivolts até algumas dezenas de milivolts. Em alguns casos, na presença de bons condutores em subsuperfície, esta tensão pode atingir algumas centenas de milivolts (Telford et al.,1990; Orellana, 1972).

Em se tratando de prospecção mineral, as anomalias SP, geralmente negativas, são relacionadas à presença de condutores, sulfetos maciços, no caso. O fenômeno, neste caso, é explicado por reações eletroquímicas na interface corpo/rocha encaixante nos níveis acima e abaixo do nível freático. A origem do conjunto destas reações é baseada nas diferenças de potencial redox nestes dois níveis separados pelo nível d'água (Sato \& Mooney, 1960).

O método do potencial espontâneo (SP), em casos ambientais ou de engenharia, tem suas principais aplicações no estudo dos movimentos da água em subsuperfície. O potencial natural ou espontâneo (SP) é causado por atividade eletroquímica ou mecânica. A 
água subterrânea é o agente mais importante no mecanismo de geração de SP. Os potenciais podem estar associados à presença de corpos metálicos, fluxo de fluidos, de calor ou de íons, contatos entre rochas de diferentes propriedades elétricas (principalmente condutividade), atividade bioelétrica de materiais orgânicos, corrosão, gradientes térmicos e de pressão nos fluidos de subsuperfície, entre outros. O estudo do SP tem sido utilizado para localizar e delinear as anomalias, bem como as fontes associadas.

\section{ELETRORRESISTIVIDADE}

\section{Resistividade Aparente (pa)}

A resistividade aparente é o parâmetro fornecido pelo método da eletrorresistividade. Seu princípio é o seguinte: quando uma corrente de intensidade I é injetada no solo graças a um emissor E (baterias ou grupo-gerador) ligado a um par de eletrodos A e B por intermédio de cabos elétricos isolados, pode-se medir uma diferença de potencial DV entre dois eletrodos M e N. A resistividade aparente ( $\rho$ a) é dada pela Eq.(1)

$$
\rho_{a}=K \frac{\Delta V}{I}
$$

onde $\mathrm{K}$ é um coeficiente que depende das dimensões e arranjo do quadripolo AMNB e é definido na Eq.(2). A Fig. 2 ilustra o princípio do método da eletrorresistividade.

$$
K=2 \pi\left(\frac{1}{A M}-\frac{1}{B M} \frac{1}{A N}+\frac{1}{B N}\right)^{-1}
$$

onde $\mathrm{AM}, \mathrm{AN}, \mathrm{BM}$ e $\mathrm{BN}$ são as distâncias entre eletrodos.

\section{Técnicas de Campo Empregadas}

São diversas as técnicas de campo para os levantamentos de eletrorresistividade. Neste trabalho foi usada a do caminhamento elétrico.

Trata-se de uma técnica geofísica de investigação horizontal a uma ou várias profundidades aproximadamente constantes, a partir de medidas tomadas na superfície do terreno.

As investigações pelo caminhamento elétrico (CE) geralmente são efetuadas ao longo de perfis, e os resultados obtidos se relacionam entre si através ou de um estudo em planta a uma profundidade determinada ou de seções com várias profundidades de investigação.

Os arranjos de campo para a execução da técnica do CE são os mais variados possíveis. Aqui serão descritos os arranjos utilizados neste trabalho, que foram o dipolo-dipolo e o gradiente. A escolha destes arranjos em particular deveu-se às características do objeto principal do trabalho, qual seja a de se determinar a abrangência em planta da região afetada pelo incidente.

\section{Arranjo Dipolo-dipolo}

Neste arranjo, os eletrodos de corrente A e B e os eletrodos $\mathrm{M}$ e $\mathrm{N}$ de potencial ou de recepção são alinhados sobre um mesmo perfil. $\mathrm{O}$ arranjo é definido pelos espaçamentos $\mathrm{X}=\mathrm{AB}=\mathrm{MN}$. A profundidade de investigação cresce com $\mathrm{R}$ e teoricamente corresponde a 1/2 R (Bertin \& Loeb, 1976) (Fig. 3).

Comumente, as medidas são feitas em várias profundidades de investigação, isto é, $n=1,2,3, \ldots$ e interpoladas, gerando uma pseudo-seção de resistividade aparente. $\mathrm{O}$ fator geométrico do arranjo



Figura 2 - Princípio do método da eletrorresistividade.M

Figure 2 - Principle of the electrical resistivity method. 
dipolo-dipolo, derivado da fórmula geral (2) pode ser generalizado para:

$$
K=2 \pi G x
$$

em que:

$$
G=\left(\frac{1}{n}-\frac{1}{n+1}+\frac{1}{n+2}\right)^{-1}
$$

e

$$
\mathrm{n}=1,2,3 \ldots
$$

\section{Arranjo Gradiente}

O arranjo gradiente (Fig. 4) consiste de uma linha de transmissão de corrente $\mathrm{AB}$ fixa, com medidas efetuadas entre os eletrodos $\mathrm{MN}$, que são deslocados sobre perfis paralelos à linha $\mathrm{AB}$.

A profundidade de investigação cresce com L e é sempre possível realizar vários gradientes simultaneamente, superpostos, com diferentes extensões de linha AB.

As estações de medida permanecem no interior de um retângulo central, com centro coincidente com o meio de $\mathrm{AB}$ e o lado menor não ultrapassa $\mathrm{AB} / 2 \mathrm{ou}$ $A B / 3$. Assume-se que na porção $1 / 3$ central de $A B$ o campo elétrico seja uniforme e não varie a profundidade investigada.

Os dados são apresentados em perfis justapostos e/ou mapas de contorno. As anomalias podem ser interpretadas quanto à posição com boa resolução lateral.

\section{POLARIZAÇÃO INDUZIDA}

Historicamente, o método da polarização induzida (IP) tem sido usado basicamente na prospecção mineral, na pesquisa de sulfetos metálicos ou mineralizações associadas.

No entanto, a utilização deste método nos últimos anos vem sendo incrementada em estudos ligados à prospecção de hidrocarbonetos, obras de engenharia, pesquisa de água subterrânea e em questões ambientais.

As técnicas e arranjos eletródicos utilizados nos levantamentos IP são aqueles usados nos levantamentos de eletrorresistividade. Ademais, os equipamentos usados na execução de ensaios IP, via de regra, também medem a eletrorresistividade, juntamente com o potencial espontâneo.

Os fenômenos físico-químicos que poderiam explicar a polarização induzida são complexos e ainda não são muito bem conhecidos. A maior parte dos autores concorda em distinguir duas origens possíveis para a polarização induzida, como descrito a seguir:

a) polarização eletrônica ou de eletrodo (entendase por eletrodo a partícula metálica ou grão mineral metálico): o fenômeno IP se manifesta com maior intensidade na presença de corpos minerais metálicos que conduzem a corrente eletronicamente. Esta polarização pode ser entendida como voltagem acumulada na interface entre o grão metálico e a solução eletrolítica que preenche os poros da rocha hospedeira. Ela ocorre porque o fluxo de corrente é muito mais lento na solução do que no metal;

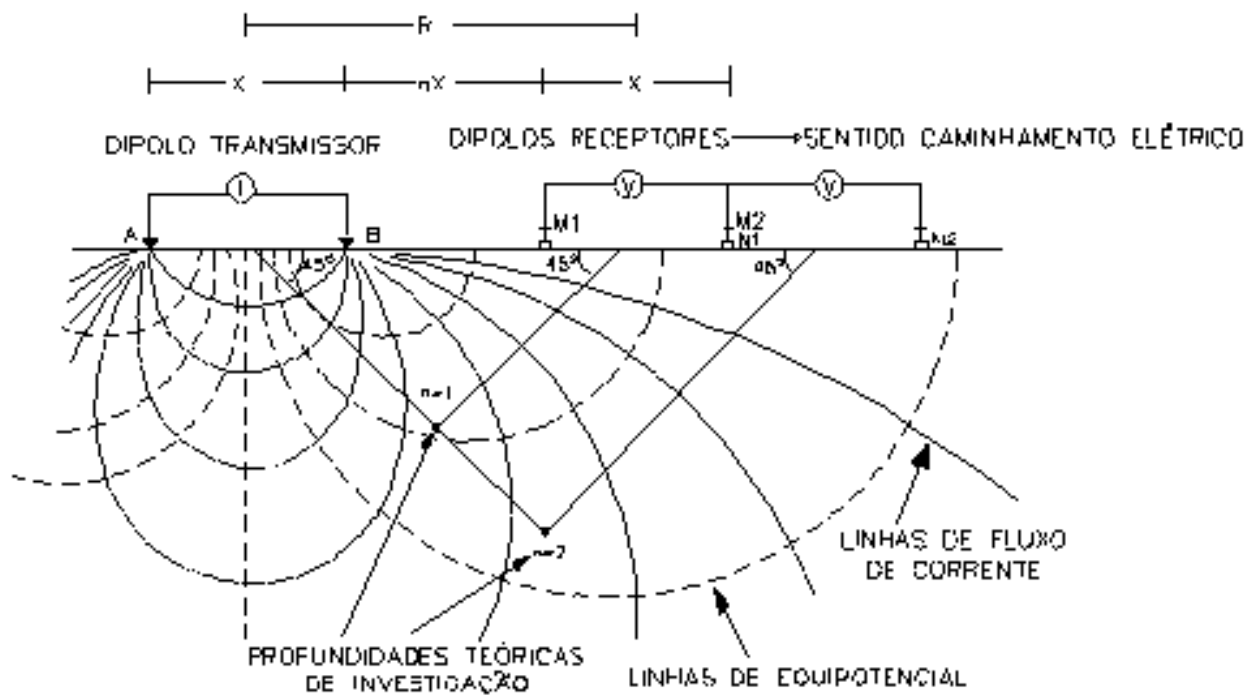

Figura 3 - Caminhamento IP-Resistividade / Disposição no campo do arranjo dipolo-dipolo.

Figure 3 - IP-resistivity profiling / dipole-dipole field array configuration. 


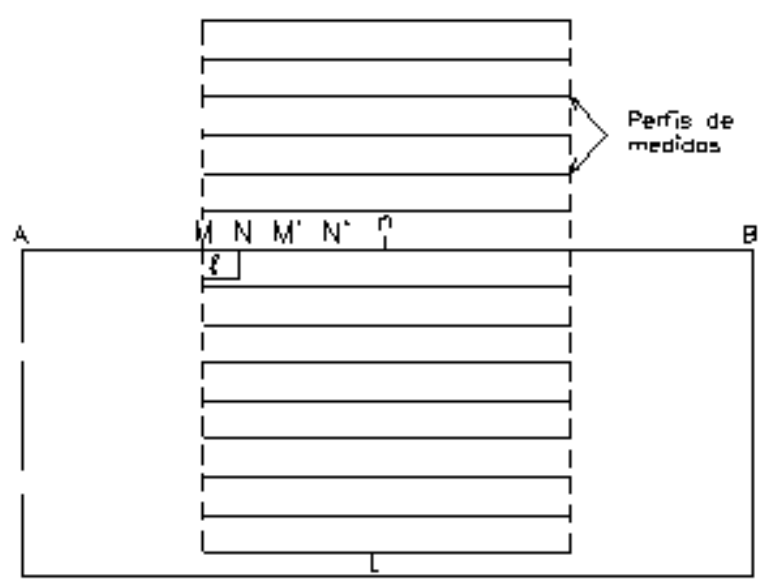

Figura 4 - Arranjo gradiente.

Figure 4 - Gradient array.

b) polarização de membrana: surge principalmente em rochas (ou subsolo em geral) em que partículas de argila bloqueiam parcialmente os caminhos da solução iônica. Quando um potencial elétrico é aplicado, os íons transportadores de cargas positivas passam através da nuvem de cátions, mas os transportadores de cargas negativas acumulam-se; formando-se, portanto, uma membrana seletiva de íons. Conseqüentemente, ocorre um excesso de cátions de um lado da membrana e, de ânions, no outro. Os gradientes de concentração de íons se opõem ao fluxo de corrente, reduzindo a mobilidade global dos íons. A polarização resultante é, em parte, função da diferença de mobilidade entre cátions e ânions.

O método IP tem sido utilizado, sem muita distinção, nas técnicas do domínio do tempo e da freqüência, e numa série de diferentes arranjos eletródicos, dos quais o mais popular seja talvez o arranjo dipolo-dipolo com vários níveis de investigação. Neste trabalho a técnica utilizada foi a do domínio do tempo, descrita sucintamente a seguir e ilustrada na Fig. 5.

O parâmetro medido no domínio do tempo é a cargabilidade aparente, obtida pela integração ao longo de um certo intervalo de tempo, da área sob a curva do transiente do potencial elétrico secundário, após o corte de um pulso de corrente, no processo de descarga, no qual a terra energizada volta ao seu equilíbrio de cargas ou ao seu estado de potencial natural. A cargabilidade aparente assim medida entre os tempos t1 e t2, normalizada pela voltagem primária $V p$, é dada por:

$$
M=\frac{1}{V p} \int_{t 1}^{t 2} V_{I P} d t
$$

Nesta equação, a unidade é milivolt.segundo por volt, quando $\mathrm{V}_{\mathrm{IP}}$ é dado em milivolts e $\mathrm{Vp}$ em volts; acrescentando-se uma normalização pelo período de integração $\Delta \mathrm{t}$, M é expressa em milivolts por volt, ou tomando-se as voltagens primária e secundária nas mesmas unidades, pode-se ter a cargabilidade em milissegundos. O que se mede é uma cargabilidade aparente Ma por ser uma resposta volumétrica de uma porção da Terra por onde a corrente transmitida se propaga com densidade maior.

\section{METODOLOGIA E TRABALHOS REALIZADOS}

Os equipamentos utilizados para os levantamentos foram transmissor TSQ3-KW e receptor IPR-10A de fabricação canadense Scintrex. Os ensaios foram executados empregando-se o domínio do tempo com pulsos de 2 segundos $\left(2 \mathrm{~s} \mathrm{ON}^{+}, 2 \mathrm{~s}\right.$ OFF, $\left.2 \mathrm{~s} \mathrm{ON}^{-} \ldots\right)$.



Figura 5 - IP domínio do tempo, curva de descarga IP (linha tracejada) e forma de onda da voltagem primária aplicada.

Figure 5 - IP time domain, IP discharge curve (dashed line) and applied primary voltage waveform.

Foram efetuados 2 arranjos gradiente com aberturas $\mathrm{AB}$ de 50 e $60 \mathrm{~m}$ e $\mathrm{MN}$ de $2 \mathrm{~m}$, sendo as medidas tomadas em malha regular $2 \times 2 \mathrm{~m}$. Também foram levantadas 2 linhas com caminhamento dipolodipolo com aberturas $\mathrm{AB}=\mathrm{MN}=6 \mathrm{~m}$ e 5 níveis teóricos de investigação, que, de acordo com o sistema de plotagem convencional são $\mathrm{n} 1=6 \mathrm{~m} ; \mathrm{n} 2=9 \mathrm{~m} ; \mathrm{n} 3=12 \mathrm{~m}$; $\mathrm{n} 4=15 \mathrm{~m}$ e $\mathrm{n} 5=18 \mathrm{~m}$. Segundo Edwards (1977) e Barker (1989), as profundidades efetivas de investigação são da ordem da metade das estimadas acima, sendo $\mathrm{n} 1=2,5 \mathrm{~m} ; \mathrm{n} 2=4,2 \mathrm{~m} ; \mathrm{n} 3=5,8 \mathrm{~m} ; \mathrm{n} 4=7,3 \mathrm{~m}$ e $\mathrm{n} 5=8,9 \mathrm{~m}$. Na malha utilizada para o arranjo gradiente foi efetuado o levantamento de potencial espontâneo (SP).

Os resultados obtidos com o arranjo dipolo-dipolo são apresentados sob a forma de pseudo-seções de resistividades e cargabilidades aparentes segundo o sistema convencional e modificadas segundo Edwards 
(1977). Os dados provenientes do arranjo gradiente são apresentados em blocos diagrama. Da mesma forma é feita a apresentação do levantamento SP.

\section{ANÁLISE DOS RESULTADOS}

A perspectiva 3D mostrada na Fig. 6 é resultado da média aritmética dos dados de resistividade dos gradientes de aberturas $A B=50 \mathrm{~m}$ e $A B=60 \mathrm{~m}$ e interpolados. $\mathrm{O}$ cálculo da malha para a interpolação dos dados foi feito com o programa aplicativo Surfer pelo método da krigagem. A perspectiva 3D mostrada na Fig. 7 é um processamento dos dados, também pelo Surfer, da malha anterior (krigagem) subtraído de outra malha, dos mesmos dados, calculado por regressão polinomial da forma $\mathrm{Z}(\mathrm{x}, \mathrm{y})=\mathrm{a}+\mathrm{bx}+\mathrm{cy}+\mathrm{dx}^{2}+\mathrm{exy}+\mathrm{fy}^{2}$, da seguinte maneira:

$$
\text { Fig. } 7=[(\operatorname{gradAB} 50+\operatorname{gradAB} 60) / 2]-Z(x, y)
$$

onde $\operatorname{grad} \mathrm{AB} 50=$ resistividade do gradiente de $\mathrm{AB}=50 \mathrm{~m}$ grad $\mathrm{AB} 60=$ resistividade do gradiente de $\mathrm{AB}=60 \mathrm{~m}$ $\mathrm{Z}(\mathrm{x}, \mathrm{y})=$ valor medido de resistividade nas coordenadas $\mathrm{X}$ e $\mathrm{Y}$ do gradiente.

Tal procedimento foi realizado visando uma melhor visualização da área afetada.

A Fig. 8 exibe a perspectiva 3D do levantamento IP do arranjo gradiente originados dos dados dos gradientes de $\mathrm{AB}=50 \mathrm{~m}$ e $\mathrm{AB}=60 \mathrm{~m}$, da seguinte forma:

Fig. $8=[\operatorname{gradAB} 50 \mathrm{~m}+\operatorname{gradAB} 60 \mathrm{~m}] / 2$.

Esta forma de apresentação dos dados foi adotada uma vez que os resultados individuais dos gradientes são similares.

A Fig. 9 contém os dados de potencial espontâneo (SP) submetidos a uma filtragem passa-banda com uma janela abrangendo 4 pontos.

As Figs. 10 e 11 mostram as pseudo-seções de resistividade e cargabilidades aparentes das linhas $1019+14,35 \mathrm{~m}$ e $1019+18,35 \mathrm{~m}$.

As perspectivas $3 \mathrm{D}$ de resistividade aparente do arranjo gradiente - Figs. 6 e 7 - apresentaram uma boa definição da área do colapso, conforme é assinalado pelos valores de resistividade mais baixos, indicados pelo rebaixamento do relevo das perspectivas. A correlação dos valores mais baixos de resistividade com o incidente é devido ao fato de que este ocasionou uma maior permeabilidade e conseqüente aumento da saturação no local. Além disso, é possível interpretar que a região esquerda da perspectiva - do ponto $12 \mathrm{~m}$ eixo y e em direção à Av. 23 de Maio, abrangendo uma área de aproximadamente $3 \times 5 \mathrm{~m}$, conforme indicado nas Figs. 6 a 9 - foi afetada pelo colapso, apresentando valores de resistividade semelhantes aos do local comprovadamente atingido.

A visualização da perspectiva 3D de cargabilidade aparente apresentado na Fig. 8, mostra que nos mesmos locais anômalos indicados nas perspectivas de resistividade, é constatado um aumento do padrão de cargabilidade, sendo possível correlacionar-se da mesma forma uma associação com a região afetada pelo colapso ocorrido.

A última perspectiva 3D, Fig. 9, é a que contém os dados de SP. Esta perspectiva mostra com bastante clareza uma feição circular na porção central do mesmo, que engloba o local do incidente. Esta feição provavelmente é devida também à alteração nos conteúdos de água do subsolo, ocasionada pelas modificações nas condições de permeabilidades e porosidades causadas pelo incidente, bem como pelas injeções de calda de cimento feitas no local.

As pseudo-seções de cargabilidade e resistividade aparentes contidas nas Figs. 10 e 11, trazem os resultados obtidos com o arranjo dipolo-dipolo efetuado nas linhas $1019+14,35 \mathrm{~m}$ e $1019+18,35 \mathrm{~m}$, respectivamente. As feições mostradas pelo dispositivo gradiente são confirmadas aqui, porém sem a mesma clareza e definição do arranjo anterior.

A profundidade de investigação atingida pelo dipolo-dipolo é da ordem de $9 \mathrm{~m}$, segundo a pseudoseção modificada de Edwards (1977). Se for considerado que foi atingida a profundidade da plotagem convencional, então a profundidade investigada será em torno de $18 \mathrm{~m}$. Para efeitos comparativos, as pseudoseções são apresentadas sob os dois aspectos, nas Figs. 10 e 11. Em ambos os casos, é possível indicar, principalmente na linha $1019+18,35 \mathrm{~m}$, uma fraca feição anômala situada entre os pontos 4 e $10 \mathrm{~m}$. A posição no perfil geológico onde ocorreu o desplacamento é a camada de areia fina siltosa, de espessura de $4 \mathrm{~m}$, cujo topo situa-se a $10 \mathrm{~m}$ de profundidade. Assim, se considerarmos a modificação de Edwards (1977), então a anomalia deve ser reflexo do incidente nas camadas superiores. No entanto, se for considerado o sistema de plotagem convencional, a anomalia é causada diretamente pelo desplacamento na camada arenosa.

No caso do arranjo gradiente, pode-se considerar que o mesmo investiga uma profundidade entre 0,1 a 0,25 vezes a abertura AB. Para Edwards (1977) a profundidade de investigação alcançada pelo gradiente vai de 0,103 a 0,192 de AB. Desse modo, sob qualquer hipótese, a porção investigada pelo gradiente 


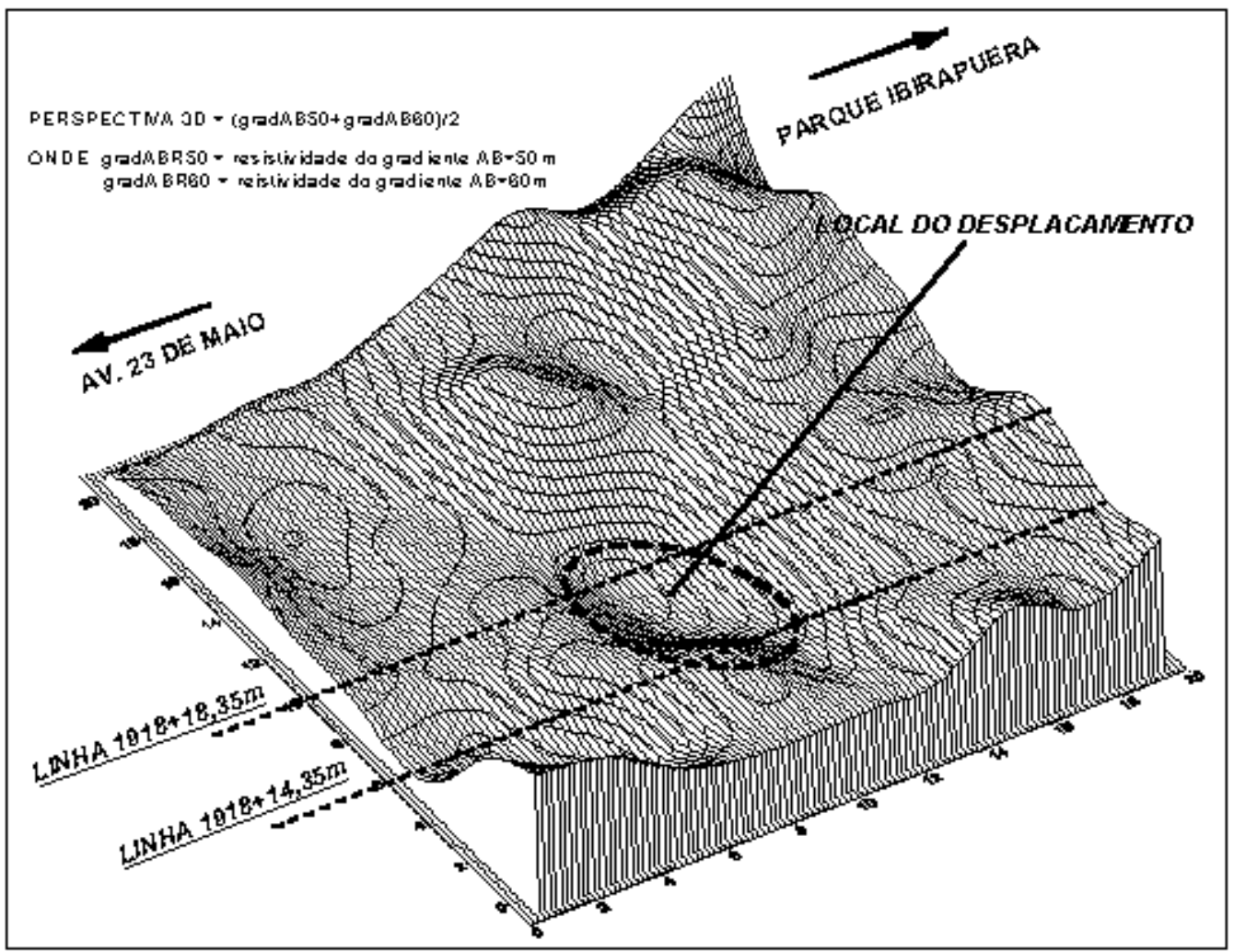

Figura 6 - Plotagem da malha de perspectiva $3 \mathrm{D}$ da resistividade do gradiente $([\operatorname{grad} A B=50+\operatorname{grad} A B=60] / 2)$.

Figure $6-3 D$ perspective mesh plot of the resistivity gradient array $([\operatorname{grad} A B=50+$ gradAB $=60] / 2)$.

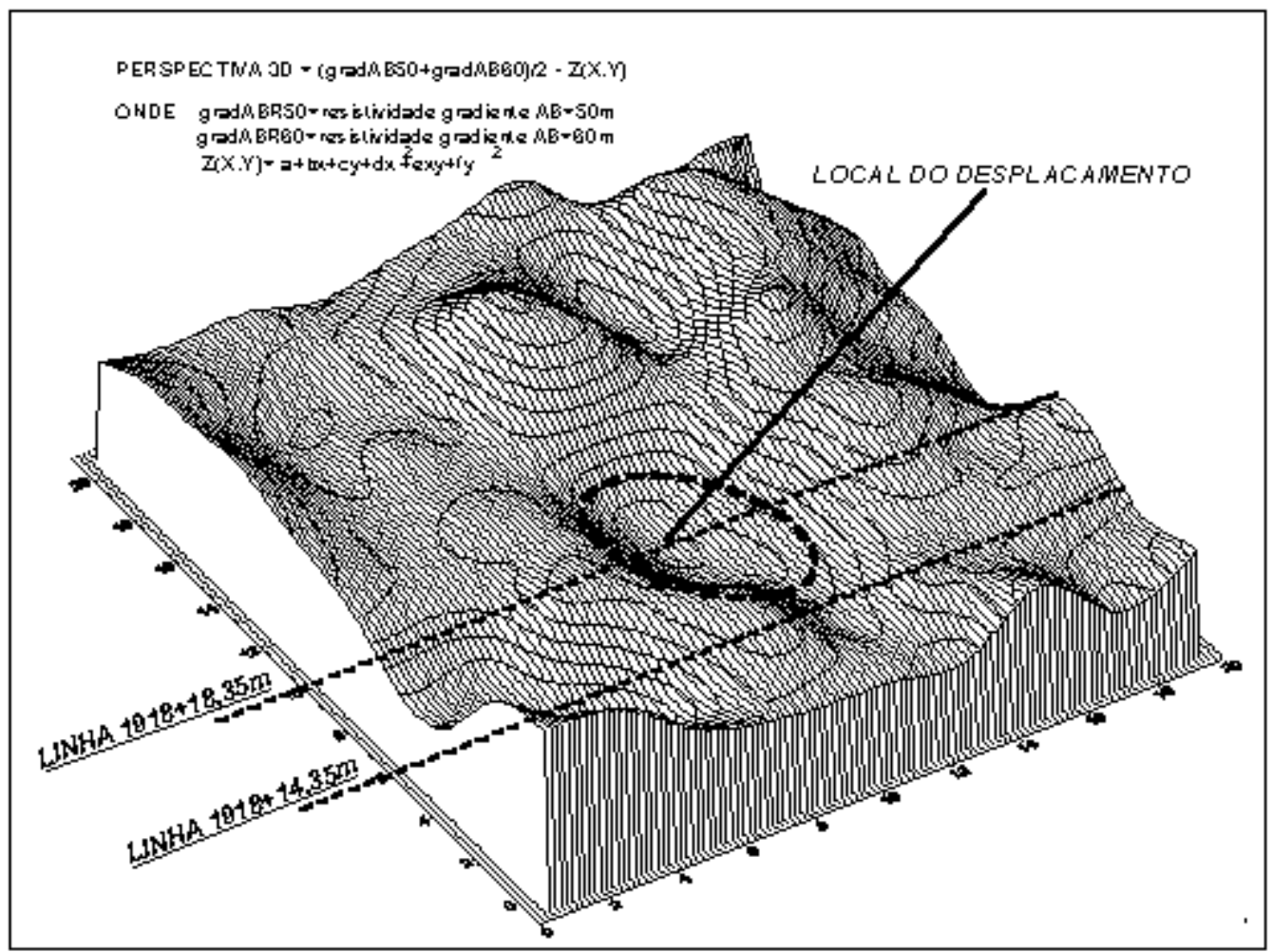

Figura 7 - Plotagem da malha de perspectiva 3D de resistividade ( $\operatorname{gradAB} 50+\operatorname{gradAB} 60-\mathrm{Z}(\mathrm{X}, \mathrm{Y})$.

Figure $7-3 D$ perspective mesh plot of the resistivity (gradAB50+gradAB60-Z(X,Y). 


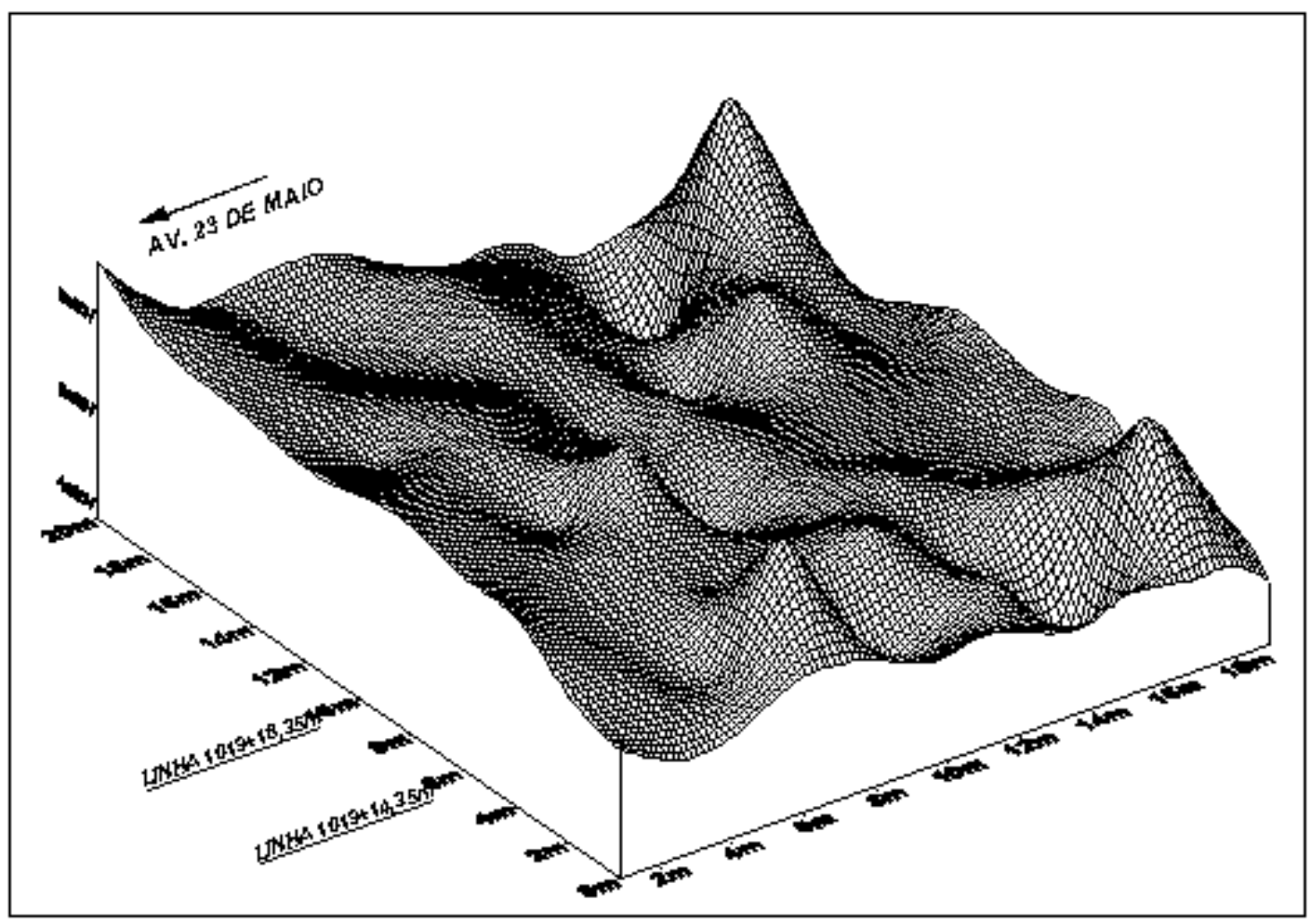

Figura 8 - Plotagem da malha de perspectiva $3 \mathrm{D}$ de cargabilidade dos gradientes $[\operatorname{gradAB}=50 \mathrm{~m}+\operatorname{gradAB}=60 \mathrm{~m}] / 2$.

Figure $8-3 D$ perspective mesh plot of the chargeability gradient array [gradAB $=50 \mathrm{~m}+\mathrm{grad} A B=60 \mathrm{~m}] / 2$.

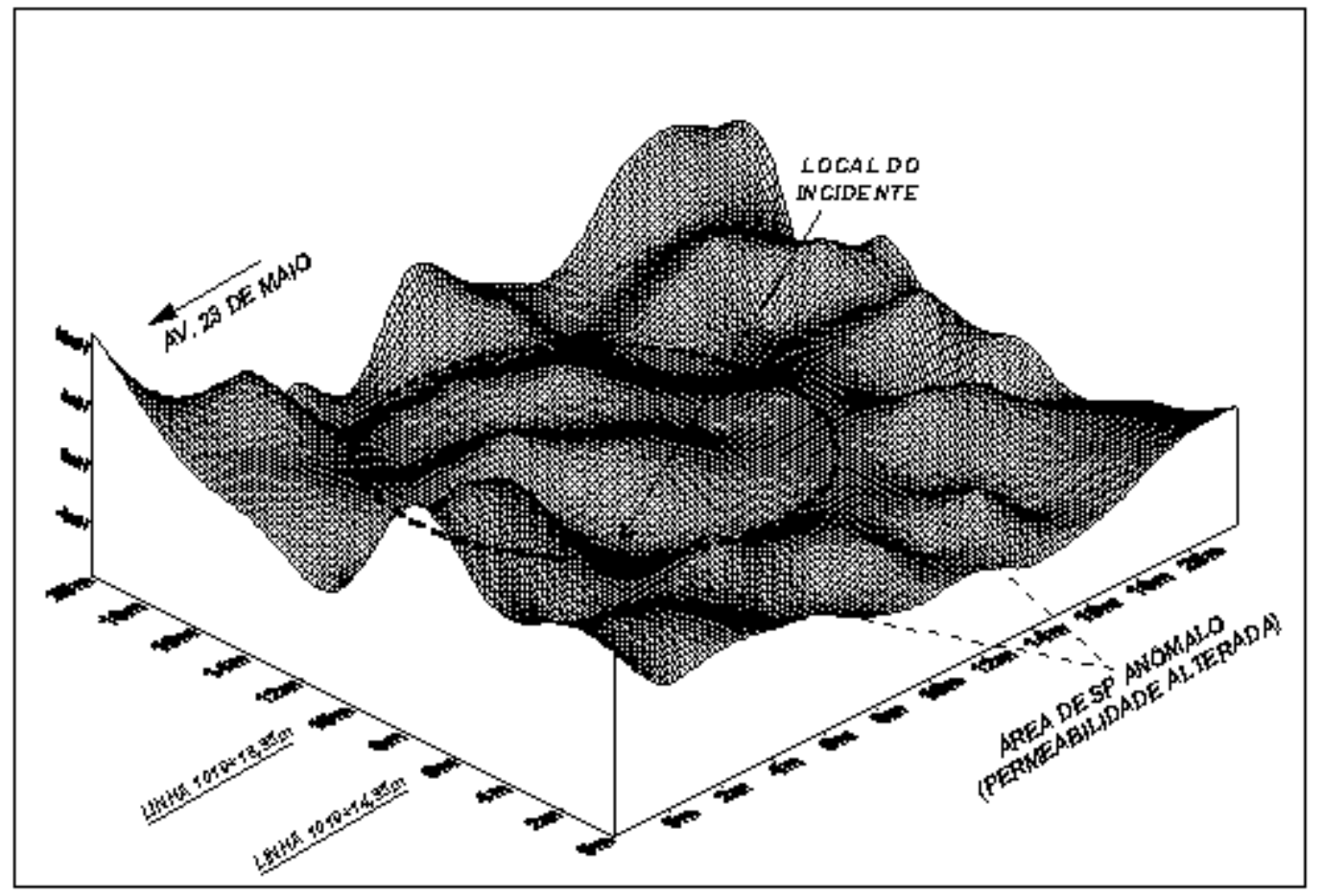

Figura 9 - Perspectiva 3D de SP, com dados filtrados.

Figure 9-3D perspective of $S P$, with filtered data. 
seguramente compreende o pacote de interesse, ou seja, a cota do acidente.

Comparando os dois arranjos empregados, cabem as considerações que seguem.

É possível aumentar ou diminuir a profundidade de investigação do arranjo gradiente sem perda do nível de detalhe da definição lateral de uma anomalia. No presente caso, a abertura entre eletrodos de medidas foi de $2 \mathrm{~m}$.

De acordo com o observado nas duas formas de plotagem das pseudo-seções e observando-se o perfil geológico-geotécnico do local (Fig. 1), a profundidade mais realística, para este caso, efetivamente atingida é aquela mostrada pela plotagem convencional.

Se for aumentado o nível de resolução do dispositivo dipolo-dipolo, tem-se como conseqüência, uma significativa perda da penetração. $O$ detalhe neste levantamento foi de $6 \mathrm{~m}$ entre medidas $(\mathrm{AB}=\mathrm{MN}=6 \mathrm{~m})$.

$\mathrm{O}$ arranjo dipolo-dipolo fornece maior detalhe de informações quanto às variações verticais dos parâmetros medidos, enquanto que o gradiente fornece uma melhor resolução nas heterogeneidades laterais destes parâmetros.

Considerando as já citadas adversidades das condições urbanas, é necessário que se procure sempre trabalhar com a relação sinal ruído mais favorável possível. Para as aberturas entre eletrodos usadas nos dois arranjos empregados, evidencia-se que a relação mais favorável é a do gradiente. Além disso, tendo em vista os objetivos a serem atingidos, ou seja, a priorização de uma definição lateral da anomalia mesmo em detrimento de uma resolução vertical e limitações físicas de espaço para os trabalhos, neste caso mostrou-se mais eficaz o arranjo gradiente.

\section{CONCLUSÕES}

Os resultados obtidos através dos levantamentos mostraram que o objetivo proposto foi atingido, qual seja o de delimitar a zona afetada pelo colapso.

A área estimada, de forma conservadora, pelos levantamentos geofísicos permitiu interpretar que a porção do terreno que sofreu as conseqüências do acidente teria uma extensão da ordem de 3 x $5 \mathrm{~m}$, no máximo, verificando-se posteriormente, através da observação direta por ocasião da retomada da obra, que a mesma abrangeu uma área de $3 \times 3 \mathrm{~m}$.

Vale lembrar que o tempo gasto para a execução dos ensaios geofísicos, realizados em caráter emergencial durante um fim-de-semana, foi de apenas dois dias, incluindo-se aí o pré-processamento dos dados, que prontamente identificou a extensão do acidente de forma preliminar.

O levantamento SP mostrou boa definição no sentido de mapear a porção do terreno afetada nos parâmetros porosidades/permeabilidades.

A Polarização Induzida indicou que as porções do terreno afetadas pelo colapso apresentam valores de cargabilidade aparentes mais elevados. As medidas de resistividade indicaram e mapearam o local do colapso e as imediações atingidas pelo mesmo, através de valores mais baixos de resistividade aparente.

Todos os três métodos aqui discutidos (Resistividade, SP e IP) mostraram resultados práticos significativos, revelando-se ferramentas valiosas e eficazes no apoio a obras civis desta natureza. O parâmetro resistividade com o emprego do arranjo gradiente mostrou, neste caso particular, uma melhor adequação/resolução de acordo com os objetivos que se buscavam, apresentando melhores resultados que o dispositivo dipolo-dipolo.

Da mesma forma, a maneira de apresentação dos dados das pseudo-seções no modo convencional, comparativamente à de Edwards (1977), quanto às profundidades atingidas, mostrou uma melhor correlação com a seção geológico-geotécnica real.

Conclui-se também que um tratamento dos dados, conforme visto na perspectiva 3D da Fig. 7, permite uma melhor visualização da extensão e posicionamento da área afetada do que com os dados brutos apresentados na Fig. 6.

Finalmente, os levantamentos geofísicos apresentaram uma ótima correlação custo/benefício, uma vez que, com base nestes dados, houve uma redução nos custos nas obras de remediação do incidente superior a $90 \%$ da estimativa inicial. Esta redução foi devida à diminuição da área a ser remediada, através da minimização do volume de material, equipamentos, mão-de-obra e principalmente tempo.

\section{AGRADECIMENTOS}

Os autores agradecem aos revisores anônimos que contribuíram significativamente para aprimorar a clareza e precisão deste trabalho.

\section{BIBLIOGRAFIA}

Barker, R. D., 1989. Depth of investigation of collinear symmetrical four-electrode arrays. Geophysics, 54: 1031-1037. 

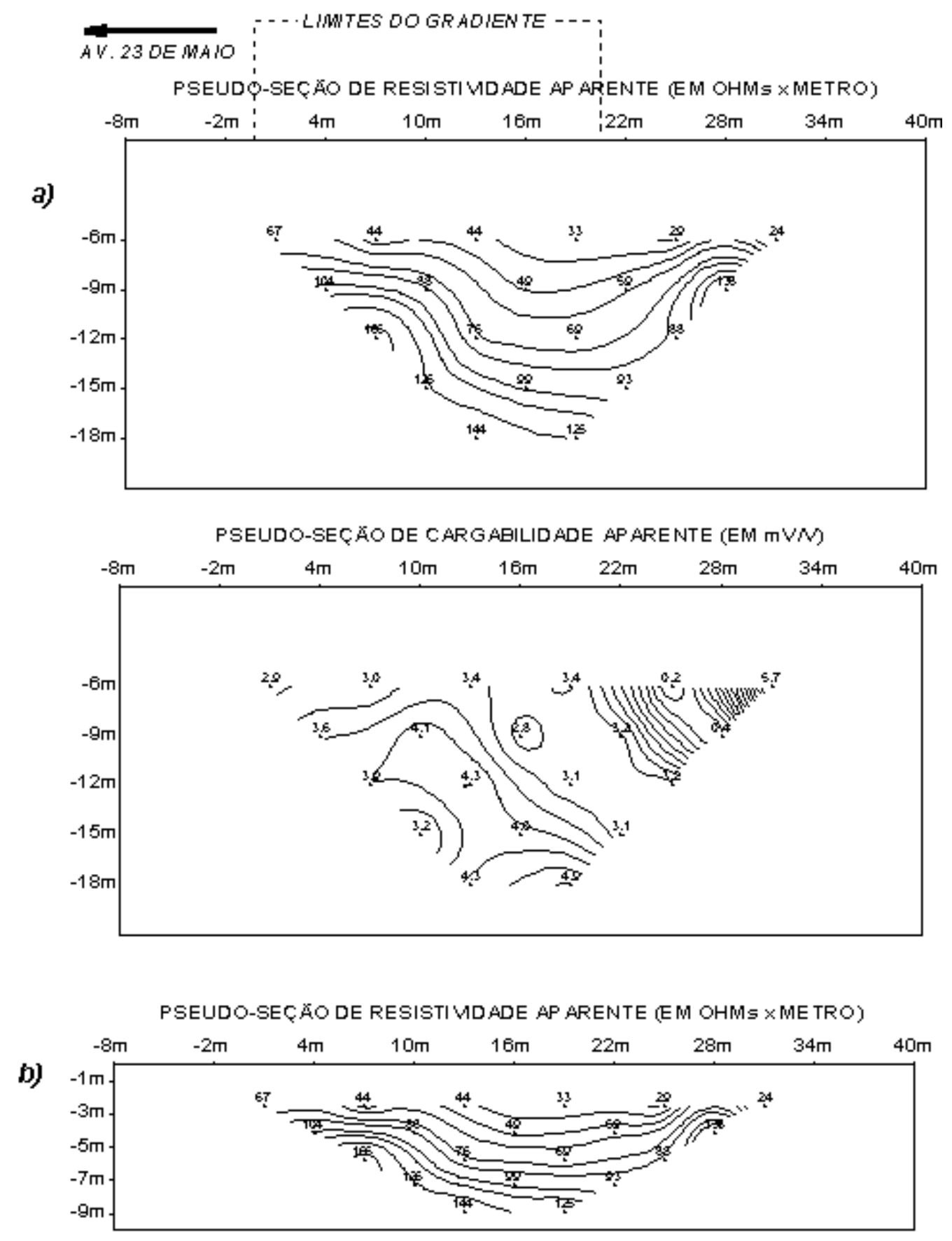

PSEUDO-SEÇÁO DE CARGABILIDADE APARENTE (EM mVN)

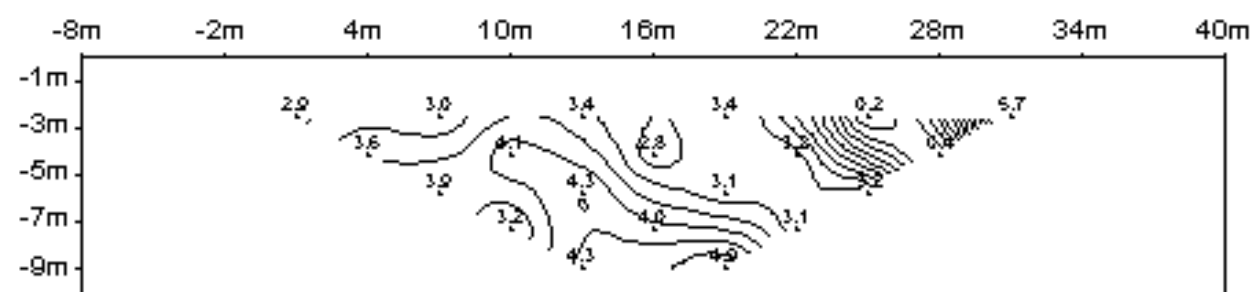

Figura 10 - Pseudo-seções de resistividade e cargabilidade da linha 1019+14,35m. a) Plotagem convencional. b) Pseudo-seção modificada segundo Edwards (1977).

Figure 10 - Resistivity and chargeability pseudosections - line 1019+14.35m. a) Conventional plot. b) Modified pseudosection, from Edwards (1977). 

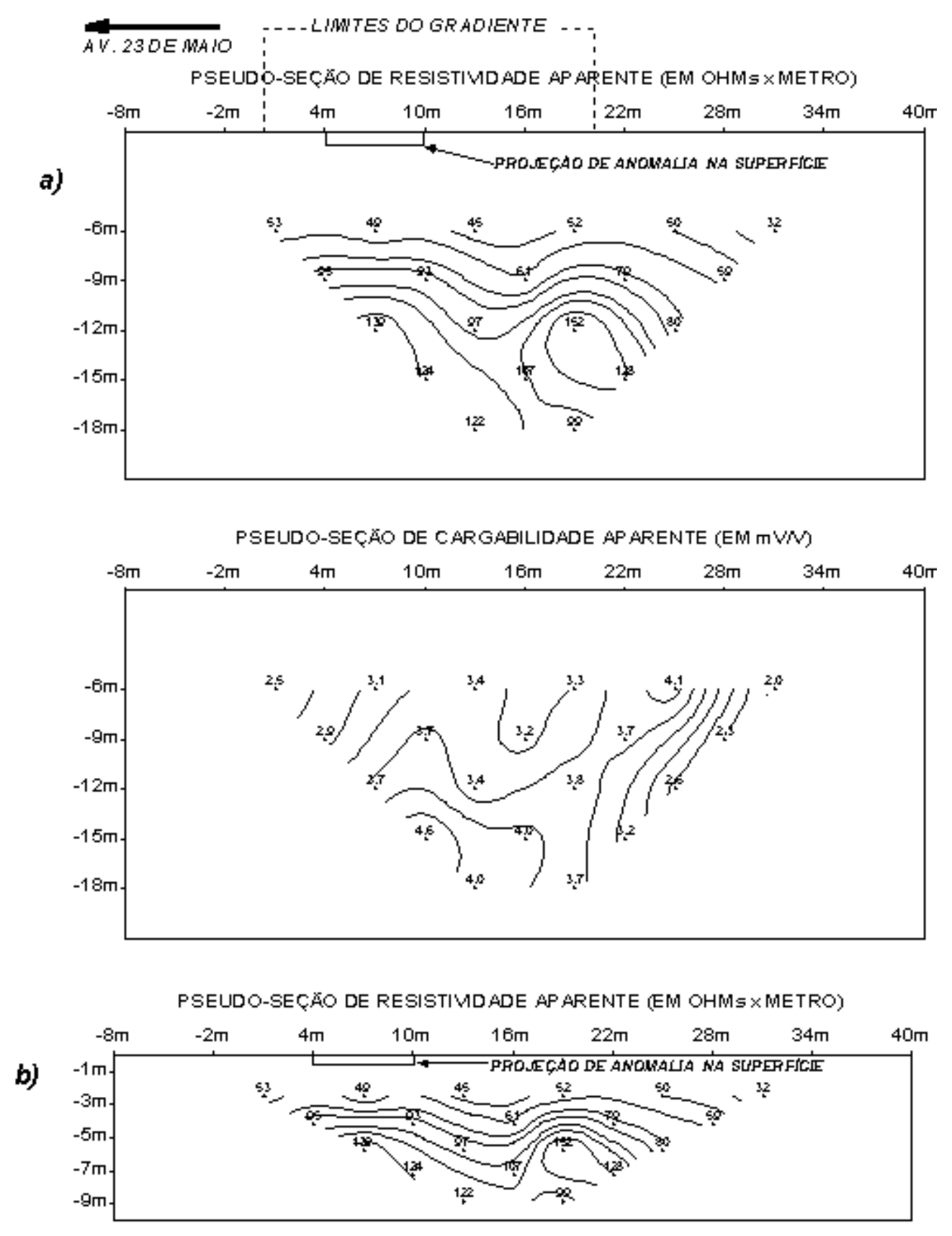

PSEUDO-SEÇAO DE CARGABILIDADE APARENTE (EM mWN)

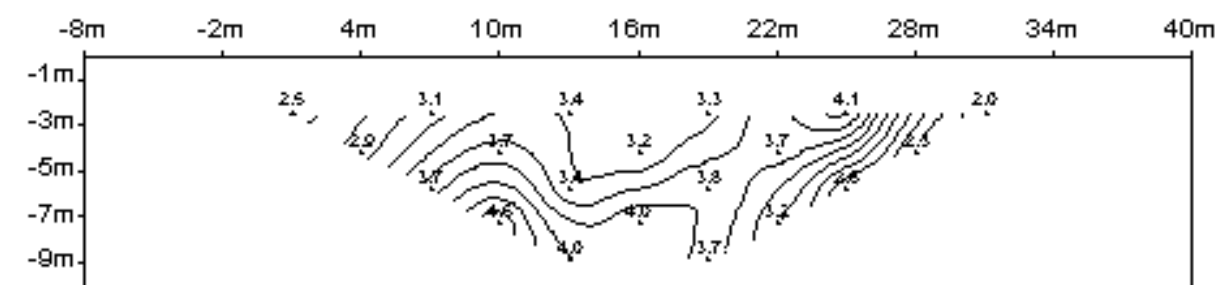

Figura 11 - Pseudo-seções de resistividade e cargabilidade da linha 1019+18,35m. a) Plotagem convencional. b) Pseudo-seção modificada segundo Edwards (1977).

Figure 11 - Resistivity and chargeability pseudosections - line 1019+18.35m. a) Conventional plot. b) Modified pseudosection, from Edwards (1977). 
Bertin, J. \& Loeb, J., 1976. Experimental and theoretical aspects of induced polarization. Gebruder Borntraeger, Berlin-Stutgart, Germany: Geopublicaton Associates, 1, 250pp.

Cozzolino, V. M. \& Martinati, L. R. \& Buono, A. V. D., 1994. Contribuição ao estudo dos movimentos tectônicos sin e pós-sedimentares na Bacia de São Paulo a partir de evidências observadas nas escavações do túnel da Eletropaulo. São Paulo. Revista Solos e Rochas, 17: 13-29.

Edwards, L. S., 1977. A modified pseudo-section for resistivity and induced-polarization. Geophysics, 42: 78-95.

Gallas, J. D. F., 1999. Geofísica - Uma Aplicação em Área Urbana de Ocorrência de Colapso de Terreno. Acta Geologica Leopoldensia, XXIII: 103-109.

Gallas, J. D. F., 2000. Principais Métodos Geoelétricos e suas Aplicações em Prospecção Mineral, Hidrogeologia, Geologia de Engenharia e Geologia Ambiental. Tese de Doutorado - Instituto de Geociências e Ciências Exatas, Universidade Estadual Paulista. Rio Claro, 174pp.

Gallas, J. D. F. \& Augusto Filho, O., 1999. Ensaios de Eletrorresistividade SP e IP em Área de Risco Associado a Colapso de Terreno. Anais VI Cong. Intern. SBGf, RJ. CD-ROM.

Gallas, J. D. F., Santoro, E., Mesquita, N. N., Diniz, H. N., Souza, L. A. P., 2000. Ensaios Geofísicos em Apoio ao Diagnóstico de Recalque de Solo em Área Urbana Ocorrido no Município de Taubaté, SP. ABES - Associação Brasileira de Engenharia Sanitária e Ambiental. Anais XXVII Cong. Interam. de Eng. Sanit. e Amb., Porto Alegre, RS. CD-ROM.
Instituto de Pesquisas Tecnológicas do Estado de São Paulo-IPT., 1996. Investigação geofísica com métodos sísmicos e elétricos em área sobrejacente ao túnel sul, alça Sena Madureira, próximo à estaca $1019+18,36 \mathrm{~m}$. Relatório IPT34.114, 33pp.

Orellana, E., 1972. Prospeccion geoeletrica en corriente continua. Madrid: Paraninfo, $523 \mathrm{p}$.

Rebouças, A. C., Riccomini, C., Ellert, N., Duarte, U., Mellito, K. M. \& Senf, L. A., 1983. Diagnóstico hidrogeológico da região metropololitana de São Paulo. Centro de Pesquisas de Águas Subterrâneas - CEPAS. Folha São Paulo 1:50.000.

Riccomini, C., 1989. O rift continental do sudeste do Brasil. Tese de Doutorado - Instituto de Geociências da Universidade de São Paulo. 256 p.

Sato, M. \& Mooney, H. M., 1960. The electrochemical mechanism of sulfide selfpotentials. Geophysics, 25: 226-249.

Taioli, F., Dourado, J. C., Gallas, J. D. F., Prado, R. L., Iyomasa, W, S. \& Cordeiro, R. P., 1997a. Geophysical Data Integration to Delineate Rupture Extension in Urban Tunnel. 10 ${ }^{\text {th }}$ SAGEEP, Reno, Nevada. 2: 835- 841.

Taioli, F., Dourado, J. C., Gallas, J. D. F., Prado, R. L., Iyomasa, W, S. \& Cordeiro, R. P., 1997b. Use of Geophysical Methods to Define the Extension of a Tunnel Roof Failure. Anais V Cong. Intern. SBGf - São Paulo, 1, pp. 421.

Takiya, H., 1991. Aplicação de métodos quantitativos espaciais a dados geolégicos da bacia de São Paulo. Dissertação de Mestrado Instituto de Geociências - Universidade de São Paulo. 109pp.

Telford, W. M., Geldart, L. P. \& Sheriff, R. E., 1990. Applied geophysics. Second Edition. Cambridge: Cambridge University Press, 770pp.

\section{SHALLOW GEOELECTRIC METHODS AND TECHNIQUES TO DEFINE AN AREA AFFECTED BY ROOF FAILURE OF ANURBANTUNNEL}

Tunnel excavation in urban areas evolves high cost and difficulties inherent to such environment. The direct investigation by boreholes is difficult due to transport and installation of the equipment. Considering indirect investigation, including geophysics, the difficulties are even greater because, in addition to those problems, the environment presents electrical, electromagnetic and seismic noises that interfere in the physical measurements. Due to this reason, geophysical studies in urban areas are not commonly reported in the literature.

This paper presents a case study where geophysics was successfully applied to solve a complex problem associated with a tunnel excavation accident in an urban area. It presents and describes the results obtained with geolectrical methods applied in an emergencial task in a risky urban area in Sao Paulo city. It also compares the 
techniques and field arrays, ways to present and interpret the data. The applied methods were electroresistivity, induced polarization (IP) and self potential (SP). The objective of the survey was to delineate the extension of a roof failure of a tunnel under excavation beneath the Ibirapuera Park in order to orientate the volume of tunnel roof should be treated. Only two days (Saturday and Sunday) were available to carry out the survey and its interpretation.

The geophysical data associated to the geologic-geotechnical information allowed estimating the accident area to $3 \times 5 \mathrm{~m}$ at maximum. After the completion of the excavation it was observed that the accident extension was of $3 \mathrm{x}$ 3 meters. The results were considered satisfactory and brought a significant economy to the final cost of the tunnel. This economy was due to the decreasing of the remediation area from the initial $20 \times 20 \mathrm{~m}$ (estimated by the engineers) to $3 \times 5 \mathrm{~m}$ (estimated by the geophysical survey).

\section{NOTES ABOUT THE AUTHORS}

\section{José Domingos Faraco Gallas}

Bacharelou-se em Geologia pela Universidade Federal do Rio Grande do Sul em 1978. Concluiu mestrado em Geofísica em 1990 pela Universidade Federal do Pará. Doutorou-se em Geociências e Meio Ambiente, ênfase em geofísica aplicada, pela Unesp - Universidade Estadual Paulista, em 2000. Foi Pesquisador do Laboratório de Geofísica Aplicada do Instituto de Pesquisas Tecnológicas do Estado de São Paulo - IPT de 1979 até início de 2002. Atualmente é Professor Adjunto do Instituto de Geociências da Universidade de São Paulo - USP. Áreas de interesse: geofísica aplicada à prospecção mineral, hidrogeologia, geologia de engenharia e geologia ambiental.

\section{Fabio Taioli}

Formou-se em Geologia pela USP em 1973. Foi geofísico da Petrobrás até 1975 quando transferiu-se para o Instituto de Pesquisas Tecnológicas do Estado de são Paulo - IPT. Obteve, em 1987, seu MSc em Mining Engineering pela The Pennsylvania State University na área de Geomecânica. Doutorou-se pelo Instituto de Geociências da USP em 1992 na área de Geofísica Aplicada. Transferiu-se para o IG-USP em 1995 onde é Professor Associado. Sua área de interesse é a aplicação de métodos geofísicos a problemas de engenharia civil e de minas e meio ambiente. É membro da SBGf, SEG, EEGS, IAEG, ISRM, ABGE, ABMS, CBMR.

\section{Walter Malagutti Filho}

É Bacharel em Geologia (1981) e Doutor em Geociências e Meio Ambiente pela Universidade Estadual Paulista - Unesp
(1991). Atualmente é professor assistente doutor de Geofísica Aplicada no Instituto de Geociências e Ciências Exatas da Unesp. Suas atividades de pesquisa concentramse na aplicação de métodos geofísicos em estudos ambientais, de engenharia, hidrogeologia e geologia regional.

\section{Renato Luiz Prado}

É Bacharel em Geologia pelo IGc-USP, 1981; Mestre em Geofísica pelo IAG-USP, 1994; Doutor em Geociências pelo IGCE-UNESP, 2000; Pesquisador do Instituto de Pesquisas Tecnológicas-IPT, 1982-2001. Professor do Departamento de Geofísica do Instituto de Astronomia, Geofísica e Ciências Atmosféricas-IAG desde 2001. Áreas de interesse: geofísica aplicada, métodos sísmicos, GPR, tratamento digital de sinais.

\section{João Carlos Dourado}

É formado em Geologia no Instituto de Geociências da Universidade de São Paulo em 1977. Completou seu doutorado em Geociências no Instituo de Geociências e Ciências Exatas da UNESP em 1997. Trabalhou no Laboratório de Geofísica do IPT (Instituto de Pesquisas Tecnológicas do Estado de São Paulo) no período de 1977 a 1997. De 1997 até hoje atua como Professor Assistente de Geofísica na Universidade Estadual Paulista (Unesp), Campus de Rio Claro, SP. 